NOTE

\title{
Granulomatous myositis associated with a novel alveolate pathogen in an adult southern leopard frog (Lithobates sphenocephalus)
}

\author{
Megan E. B. Jones ${ }^{1,4}$, Aníbal G. Armién ${ }^{2}$, Betsie B. Rothermel ${ }^{3}$, Allan P. Pessier ${ }^{1, *}$ \\ ${ }^{1}$ Amphibian Disease Laboratory, Wildlife Disease Laboratories, Institute for Conservation Research, San Diego Zoo Global, \\ PO Box 120551, San Diego, California 92112-0551, USA \\ ${ }^{2}$ Minnesota Veterinary Diagnostic Laboratory, University of Minnesota, Minnesota 55108, USA \\ ${ }^{3}$ Archbold Biological Station, Lake Placid, Florida 33862, USA
}

${ }^{4}$ Present address: Department of Pathology, College of Veterinary Medicine, University of Georgia, 501 D.W. Brooks Drive Athens, Georgia 30602, USA

\begin{abstract}
Since 1999, infections with an incompletely characterized alveolate protozoan variously reported as a Dermocystidium-like organism, a Perkinsus-like agent, and Dermomycoides sp. have been associated with mortality events in tadpoles of ranid frogs from the USA. However, disease or mortality events due to this organism have not been described in post-metamorphic animals. We describe infection with a morphologically similar protozoan presenting itself as a leg mass in a free-ranging adult southern leopard frog Lithobates sphenocephalus. Using histological examination, we found a mass within skeletal muscle; this mass was composed of macrophages with intracytoplasmic, thick-walled, 4 to $6 \mu \mathrm{m}$ in diameter, spherical basophilic protozoal organisms that exhibited green autofluorescence with epiflorescence illumination. Using transmission electron microscopy, organism cell walls were found to have electron-dense plates that, when viewed by scanning electron microscopy, were reminiscent of the thecal plates of dinoflagellates. Additional morphologic and molecular phylogenetic research is needed to resolve the taxonomic status of this organism.
\end{abstract}

KEY WORDS: Amphibian · Protozoa · Dermomycoides sp. · Ultrastructure

\section{INTRODUCTION}

Since 1999, sporadic mortality events in free-ranging tadpoles of ranid frogs in the USA have been attributed to an emerging disease caused by an incompletely characterized protistan parasite variously reported as a Dermocystidium-like organism, a Perkinsus-like agent, and Dermomycoides sp. (Green et al. 2002, 2003, Cook 2008). Analysis of a near full-length 18s rRNA sequence from southern leopard frog Lithobates sphenocephalus tadpoles suggested that these organisms were most closely related to unspecified types of alveolate protozoa discovered in freshwater lakes and marine hydrothermal vents (Davis et al. 2007). Other affected species have included the wood frog L. sylvaticus, American bullfrog $L$. catesbeianus, mink frog L. septrionalis, green frog $L$. clamitans, and the gopher frog $L$. capito. Of particular concern for amphibian conservation are outbreaks in the remnant populations of the critically endangered dusky gopher frog $L$. sevosus. Geographically, infection with the parasite is 
widely distributed, having been reported from the states of Alaska, Georgia, Florida, Maine, Mississippi, Minnesota, New Hampshire, North Carolina, and Virginia.

Mortality events caused by this organism have been described only in larvae (tadpoles), with persistence of the organism through the host's metamorphosis in some cases (Davis et al. 2007, Cook 2008). However, evidence of infection in adult frogs has been limited to observation of spores within the intestinal lumen (Cook 2008). Diseased tadpoles typically show clinical signs of lethargy and bloating, and on postmortem examination there is pronounced enlargement and white discoloration of the liver, spleen, and kidneys. On histologic examination there is massive infiltration of viscera by 6 to $9 \mu \mathrm{m}$ diameter spherical basophilic organisms with little or no associated host inflammatory response. In this report we describe infection with morphologically similar or identical protozoans that had a unique clinical presentation as a leg mass in an adult frog.

\section{MATERIALS AND METHODS}

\section{Case history}

In January 2006, an adult, male southern leopard frog Lithobates sphenocephalus was collected as part of an amphibian monitoring project at the United States Department of Energy's Savannah River site in


physical examination, there was a $3 \mathrm{~cm}$ diameter, firm, immovable, mass in the right quadriceps region (Fig. 1). The frog was euthanized by immersion in tricaine methanesulfonate (Finquel ${ }^{\circledR}$, Argent Chemical Laboratories) and preserved in 10\% neutral buffered formalin until December 2010, when a necropsy was performed.



Fig. 1. Lithobates sphenocephalus. A mass rising from the leg of an adult southern leopard frog

\section{Light microscopy}

After fixation in 10\% neutral buffered formalin, samples from the leg mass, as well as all major organs, were processed routinely for histologic examination. Histologic sections were cut at $5 \mu \mathrm{m}$ and stained with hematoxylin and eosin; selected sections were also stained with Grocott's methenamine silver (GMS), Periodic Acid-Schiff (PAS) with and without diastase, and Congo Red. Additionally, unstained sections were examined with epiflorescence illumination using a Nikon Eclipse 80i microscope. Wet mounts were prepared by scraping the formalin-fixed tissue with a new scalpel blade and mounting the resulting material in water on a clean microscope slide.

\section{Electron microscopy}

For transmission electron microscopy (TEM) formalin-fixed tissue was postfixed in $0.166 \mathrm{M}$ cacodylate-buffered, 3\% glutaraldehyde with 1\% tannic acid solution (Electron Microscopy Sciences), followed by a second postfixation treatment in $1 \%$ osmium tetroxide (Electron Microscopy Sciences). Ultrathin sections of $80 \mathrm{~nm}$ were stained with uranyl acetate and lead citrate (Electron Microscopy Sciences) and examined with a JEM 1200 EXII transmission electron microscope.

For scanning electron microscopy (SEM) tissues were dehydrated in a graded ethanol series, critical point dried under $\mathrm{CO}_{2}$, sputter coated with gold, and examined with a Hitachi S3500N scanning electron microscope.

\section{RESULTS}

On histologic examination, the mass replaced and infiltrated pre-existing skeletal muscle bundles (Fig. 2a) and was composed of sheets of presumptive macrophages with myriad intracytoplasmic, thick-walled, 4 to $6 \mu \mathrm{m}$ in diameter, spherical basophilic protozoal organisms (Fig. 2b). Occasionally, organisms were present extracellularly in skeletal muscle, and very small numbers of organisms were observed in macrophages within the kidney, spleen, and liver. Morphologic detail of the organisms was enhanced on wet mount examination (Fig. 3). Organisms were positive with GMS, PAS (with and without diastase), and Congo Red stains and exhibited diffuse bright green auto- 


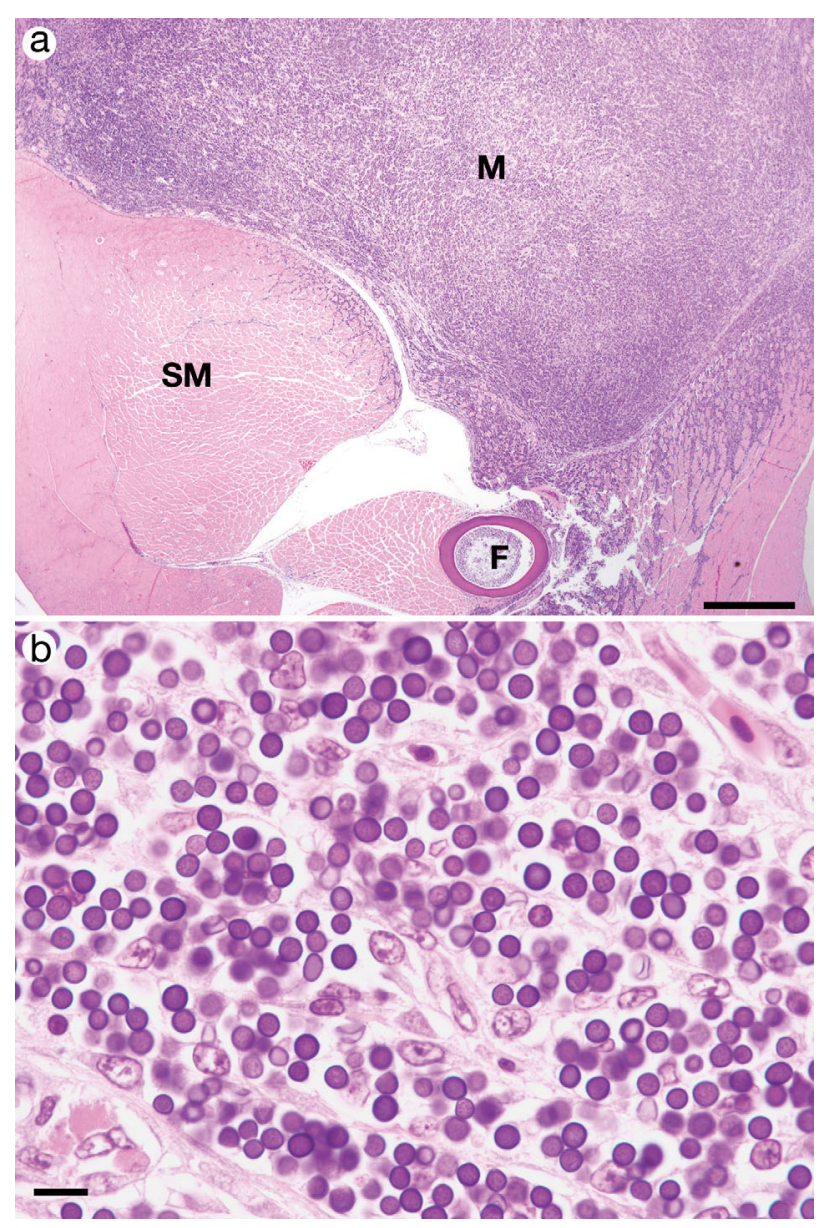

Fig. 2. Lithobates sphenocephalus. (a) Histologic cross section of the leg showing unaffected skeletal muscle (SM), the leg mass (M), and femur (F). The mass replaces and infiltrates the SM bundles. Hematoxylin and eosin stain. Scale bar $=1.2 \mathrm{~mm}$. (b) Higher magnification of the leg mass. SM fibers are replaced by presumptive macrophages with myriad intracytoplasmic spherical organisms. Hematoxylin and eosin stain. Scale bar $=14 \mu \mathrm{m}$

fluorescence when examined with epiflorescence illumination.

Using TEM, ultrastructural preservation of frog cells was poor, as anticipated, because of initial tissue fixation in formalin. Intact and degenerating organisms ranging from approximately 3.5 to $6 \mu \mathrm{m}$ in diameter were identified within macrophage cytoplasm (Fig. 4). Organisms had a 0.5 to $0.8 \mu \mathrm{m}$ thick trilaminar wall, with the middle layer composed of multiple electron-dense plates. The organism cytoplasm had numerous double-membrane-bound inclusion bodies of 0.2 to $0.7 \mu \mathrm{m}$ and occasional mitochondria with tubular cristae. Using SEM, organisms were polyhedral, with an outer surface composed of square to hexagonal plates separated by raised ridges (Fig. 5).

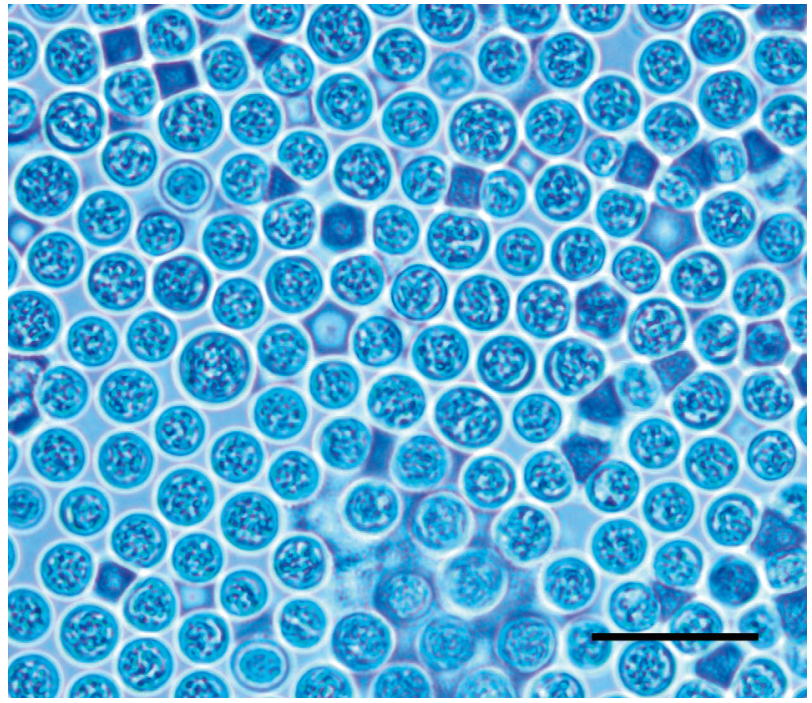

Fig. 3. Lithobates sphenocaphalus. Unstained wet mount from scrapings of the leg mass. Myriad spherical thick-walled protozoal organisms are visible. Scale bar $=12 \mu \mathrm{m}$

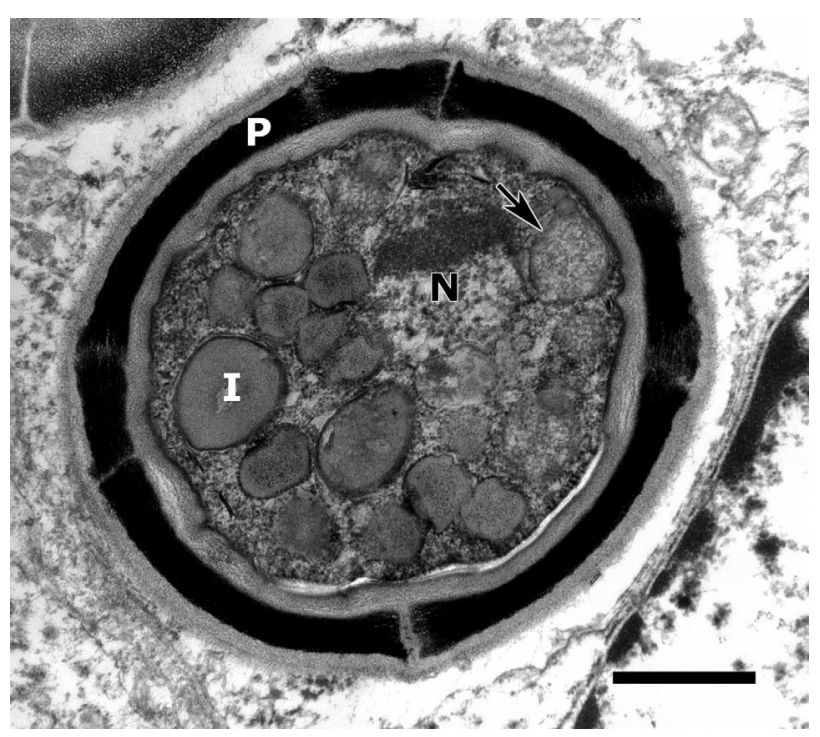

Fig. 4. Lithobates sphenocephalus. Transmission electron micrograph of an organism spore within the cytoplasm of a presumptive macrophage (frog cell nucleus is at the lower right). Features include a trilaminar cell wall with electrondense plates $(\mathrm{P})$, membrane-bound cytoplasmic inclusions (I), parasite nucleus $(\mathrm{N})$, and mitochondria with faintly visible tubular cristae (arrow). Scale bar $=1 \mu \mathrm{m}$

\section{DISCUSSION}

In this adult southern leopard frog Lithobates sphenocephalus, the spherical organisms with a polyhedral surface, as observed by histology and SEM, were indistinguishable in size and morphology from 




Fig. 5. Lithobates sphenocephalus. Scanning electron micrograph of an organism spore with a polyhedral surface composed of square to hexagonal plates. Scale bar $=2.5 \mu \mathrm{m}$

the novel alveolate pathogen reported previously to cause systemic infections in $L$. sphenocephalus tadpoles (Davis et al. 2007). The alveolate protists (superphylum Alveolata) include the phyla Ciliophora (ciliate protozoa), Dinoflagellata (dinoflagellate protozoa), Apicomplexa (e.g. the coccidian parasites) and Perkinsozoa (including Perkinsus marinus, an important pathogen of oysters) (Leander \& Keeling 2003, Leander 2008). Morphologic features of organisms in the present case that are consistent with, but not necessarily specific for, members of the Alveolata include: (1) tubular mitochondrial cristae (Leander 2008); (2) green cytoplasmic autofluorescence, as observed in some dinoflagellates and other microalgae (Tang \& Dobbs 2007); (3) positive histologic staining of organism cell walls with GMS, PAS (positive after diastase treatment indicating the absence of glycogen), and Congo Red, which is a pattern consistent with a cellulose composition; and, finally, (4) the polyhedral shape of the organisms, which is reminiscent of the thecal plates of dinoflagellates (Leander 2008).

Using light microscopy alone, the morphology and size of the spores in this case could resemble either fungal organisms or mesomycetozoan parasites of the genus Amphibiocystidium, which includes amphibian parasites previously assigned to the genera Dermocystidium, Dermosporidium, and Dermomycoides (Pascolini et al. 2003). Using TEM, the observation of tubular, rather than lamellar, mitochondrial cristae suggests that the organisms are not fungi. Furthermore, using TEM, the wall of the organism in the present case has electron-dense plates that are not present in the wall of spores from
Dermocystidium ranae (Pascolini et al. 2003). Molecular characterization of the organisms in the present case was not pursued because tissues had been subjected to prolonged $(>2 \mathrm{yr})$ storage in formalin. Clearly, additional morphologic and molecular phylogenetic work is needed to resolve the taxonomic status of this organism or potentially a group of morphologically similar organisms affecting ranid frogs in the USA.

To our knowledge, this is the first report of disease caused by this organism or similar organisms in an adult frog, and demonstrates a significantly different clinical presentation to that seen in tadpoles. It is unclear if this frog survived infection as a tadpole with persistence of infection into adulthood or if the case represents a more recent infection. Experimental studies conducted in Lithobates sphenocephalus and L. sylvatica tadpoles demonstrated transmission of infection by both ingestion of 'spores' (the spherical stage observed in histologic tissue sections) and by exposure to motile zoospores hatched from spores treated under very specific conditions of desiccation and rehydration (Cook 2008). However, in those experiments, systemic infection and disease were only observed in embryos and early-stage tadpoles ('hatchlings') exposed to the zoospore stage. Laterstage tadpoles or those exposed only to spores remained healthy, and, if infection occurred, it was limited to the intestinal lumen. Attempts to experimentally infect adult amphibians have not been reported. The organisms described in the present report were observed intracellularly, presumably within macrophages, whereas infections in tadpoles are extracellular without significant inflammation. This observation could be consistent with either a mechanism for clearance of infection acquired as a tadpole or with age-related or individual idiosyncratic differences in host response to recent infection.

In contrast to the mass mortality events described in tadpoles, disease in this adult frog seems to have been a sporadic occurrence and no similarly affected individuals were observed during the course of the field survey. In addition, to our knowledge, no mortality events in tadpoles due to alveolate-type parasites have been observed at this study site. Although the large lesion in the muscle could eventually have affected mobility or feeding behavior, at the time of collection the frog had adequate visceral fat stores and appeared to be otherwise healthy. Therefore, the significance of the alveolate infection in this case, either as a contributor to amphibian mortality or as a potential reservoir of infection at this location, is unknown. 
Acknowledgements. We thank J. R. Mendelson for bringing this unique frog to our attention, G. Graeter for capturing the frog, Y. Cates for excellent histology support, and B. Leander for initial consultation on the TEM images. This project was supported by National Leadership Grant LG-2508-0066 from the Institute for Museum and Library Services. Any views, findings, conclusions, or recommendations expressed in this publication do not necessarily represent those of the Institute of Museum and Library Services. A Leap Study award from the National Science Foundation DEB 0242874 supported field surveys that led to the discovery of the affected frog.

\section{LITERATURE CITED}

Cook JO (2008) Transmission and occurrence of Dermomycoides sp. in Rana sevosa and other ranids in the North Central Gulf of Mexico States. MS thesis, University of Southern Mississippi, Hattiesburg, MS

Davis AKM, Yabsley M, Keel K, Maerz JC (2007) Discovery of a novel alveolate pathogen affecting southern leopard frogs in Georgia: description of the disease and host effects. EcoHealth 4:310-317

Green DE, Converse KA, Schrader AK (2002) Epizootiology of sixty-four amphibian mortality events in the USA, 1996-2001. Ann NY Acad Sci 969:323-339

Green DE, Feldman SH, Wimsatt J (2003) Emergence of a Perkinsus-like agent in anuran liver during die-offs of local populations: PCR detection and phylogenetic characterization. Proc Am Assoc Zoo Vet 2003:120-121

Leander BS (2008) Alveolates. Alveolata, Version 16 September 2008. In: The tree of life web project. Available at: http://tolweb.org/Alveolates/2379/2008.09.16 (accessed 12 April 2012)

Leander BS, Keeling PJ (2003) Morphostasis in alveolate evolution. Trends Ecol Evol 18:395-402

> Pascolini R, Daszak P, Cunningham AA, Tei S and others (2003) Parasitism by Dermocystidium ranae in a population of Rana esculenta complex in central Italy and description of Amphibiocystidium n. gen. Dis Aquat Org 56:65-74

> Tang YZ, Dobbs FC (2007) Green autofluorescence in dinoflagellates, diatoms, and other microalgae and its implications for vital staining and morphological studies. Appl Environ Microbiol 73:2306-2313

Editorial responsibility: Alex Hyatt, Geelong, Victoria, Australia
Submitted: April 17, 2012; Accepted: October 1, 2012

Proofs received from author(s): November 16, 2012 